\title{
Registro de Euseius consors (Acari: Phytoseiidae) en aguacates de Michoacán, México
}

\author{
Report of Euseius consors (Acari: Phytoseiidae) \\ on avocado trees of Michoacan, Mexico
}

\author{
(D) MAYRA RAMOS-LIMA ${ }^{1}$; ID JOSÉ DE JESÚS AYALA-ORTEGA2; (DD MA \\ BLANCA NIEVES LARA-CHÁVEZ²; (iD MARGARITA VARGAS-SANDOVAL ${ }^{2}$
}

\footnotetext{
${ }^{1}$ Instituto Tecnológico Superior de Los Reyes, Michoacán, México, ramosmayra1954@gmail.com² Universidad Michoacana San Nicolás de Hidalgo, Michoacán, México,cordoba_1821@hotmail.com,Chavez12001@yahoo.com.mx, vargasmarga@hotmail.com
}

\begin{abstract}
Autor para correspondencia
Margarita Vargas Sandoval. Ph. D. en Ciencias Biológicas, Facultad de Biología, Universidad Michoacana San Nicolás de Hidalgo, Ciudad Universitaria Avenida Francisco J. Mujica s/n. C. P. 58030 Morelia, Michoacán, México, vargasmarga@hotmail.com, https://orcid. org/0000-0002-4835-8779.
\end{abstract}

\section{Citación sugerida}

RAMOS-LIMA, M.; AYALA-ORTEGA, J. J.; LARA-CHÁVEZ, M. B. N.; VARGAS-SANDOVAL, M. 2021. Registro de Euseius consors (Acari: Phytoseiidae) en aguacates de Michoacán, México. Revista Colombiana de Entomología 47 (2): e10243. https://doi.org/10.25100/ socolen.v47i2.10243

Recibido: 01-jul-2020

Aceptado: 24-dic-2020

Publicado: 28-jul-2021

Revista Colombiana de Entomología ISSN (Print): 0120-0488

ISSN (On Line): 2665-4385

https://revistacolombianaentomologia.univalle.edu.co

Open access

(c) (1) (2)(2) BY-NC-SA 4.0

Publishers: Sociedad Colombiana de Entomología SOCOLEN (Bogotá, D. C., Colombia) https://www.socolen.org.co

Universidad del Valle (Cali, Colombia)

https://www.univalle.edu.co

(C) 2021 Sociedad Colombiana de Entomología SOCOLEN y Universidad del Valle - Univalle
Resumen: Como apoyo a la descripción de la diversidad de ácaros Phytoseiidae y en consecuencia al control biológico, se informa, para México, al ácaro Euseius consors. Éste fue recolectado en plantas de aguacate (Persea americana) en siete municipios del Estado de Michoacán, asociado a Oligonychus persea (Acari: Tetranychidae), uno de los ácaros plagas más importantes en este cultivo. La presente nota es un aporte al conocimiento, base para la descripción de un potencial agente de control biológico y del servicio ecosistémico que potencialmente presta en este agroecosistema.

Palabras clave: Enemigos naturales, control biológico, ácaros depredadores, Euseius consors, Persea americana.

Abstract: To support the description of the diversity of Phytoseiidae mites and according to biological control, the mite Euseius consors is reported for Mexico. It was collected from avocado plants (Persea americana) in seven municipalities in the state of Michoacán, associated with Oligonychus persea (Acari: Tetranychidae), one of the most important pest mites in this crop. Hence, this scientific note is a contribution to the knowledge, the basis for the description of a potential biological control agent, and the ecosystem service that it can provide.

Keywords: Natural enemies, biological control, predator mites, Euseius consors, Persea americana.

\section{Introducción}

México es el principal productor de aguacate en el mundo, liderado por el estado de Michoacán con una producción de 381,733.96 t, las que representan más del $80 \%$ de la producción total nacional. En este estado, el aguacate se produce en 46 municipios donde Tancítaro es el líder productor, seguido de Tacámbaro, Salvador Escalante, Uruapan y Ario de Rosales, los que constituyen el centro de producción y es el aguacate un rubro exportable y base económica de la agricultura en este estado (Servicio de Información Agroalimentaria y Pesquera 2021).

Los ácaros Oligonychus perseae (Tuttle, Baker y Abbatiello, 1976) y Oligonychus punicae (Hirst, 1926) son plaga común en los huertos de aguacate (Equihua-Martínez et al. 2007). Las colonias pueden encontrarse tanto en el haz (O. punicae) de las hojas como en el envés (O. perseae). De forma general, los síntomas de las áreas de las hojas infestadas muestran una coloración pardo-rojiza en el haz y puntos cloróticos cuando es por el envés. En infestaciones intensas, se pueden observar las exuvias de los ácaros en la superficie foliar y en casos severos exfoliación. Dependiendo de la variedad, de las condiciones climáticas y de las labores de manejo, estos ácaros pueden ser una plaga de significativa importancia en aguacateros en el estado de Michoacán. El daño que producen puede causar bronceadura y reducción en la actividad fotosintética debido a que succionan el contenido celular de las hojas, se señalan que densidades de 300 ácaros por hoja ocasionan además del bronceado de hojas, una defoliación parcial, o la defoliación puede también ocurrir cuando se presentan 70 hembras adultas por hoja durante periodos de sequía (Peña y Wysoki 2008). 
Los ácaros de la familia Phytoseiidae son los depredadores más comunes de ácaros fitófagos (Moraes et al. 2004) y las especies presentes en aguacateros no están exentas de su actividad biorreguladora. Por lo que la identificación de las especies de esta familia es base fundamental para el establecimiento de planes de manejo de los ácaros plaga en aguacate (Estrada-Venegas et al. 2002). A la vez, los servicios relacionados con el control biológico son otro valioso aporte de los agroecosistemas, ya que dentro de los cultivos se presentan interacciones bióticas complejas, las cuales permiten que las diversas poblaciones se mantengan en niveles estables o por debajo de los umbrales de daño económico y se desarrollen actividades de parasitismo, depredación, entre otras (Corredor Camargo et al. 2012), esto puede ser de elevado valor en la regulación de plagas y la descripción de la diversidad de estos agentes de control biológico, constituye la base para el conocimiento de esos procesos.

El objetivo del presente trabajo fue identificar las especies de ácaros Phytoseiidae en árboles de aguacate en Michoacán.

\section{Materiales y Métodos}

Los ácaros fueron recolectados en siete localidades de Michoacán, México en hojas de aguacatero (Persea americana Miller variedad: Hass) junto a Oligonychus persea (Acari: Tetranychidae). En la Tabla 1, se muestran las localidades, el nombre de las huertas y la localización geográfica de las mismas.

Los ejemplares fueron colectados mediante una aguja entomológica, colocados en un portaobjeto liso sobre una gota de medio de Hoyer y cubiertos con cubreobjetos; posteriormente, se decoloraron sobre una plancha de calentamiento de laboratorio (Modelo Análogo PLA- $6003^{\circledR}$ ) a $45^{\circ} \mathrm{C}$ y se observaron de forma intermitente, hasta visualizar una transparencia total. La identificación taxonómica se realizó mediante las claves de Moraes et al. (2004) y Chant y McMurtry (2005). Se recolectó un total de 164 ejemplares, los que se encuentran en la colección de Acarología, de la Facultad de Biología de la Universidad Michoacana de San Nicolás de Hidalgo (UMSNH), Morelia, México.

\section{Resultados y discusión}

Se registró una sola especie perteneciente al género Euseius: Euseius consors (De Leon, 1962) (Acari: Phytoseiidae) y todos los ejemplares recolectados fueron hembras. De forma general, se observaron los chelíceros reducidos, placa dorsal lisa con ligeras estriaciones en la parte anterior y Z1 presente, la placa esternal muestra una proyección posterior; en la placa ventrianal en forma de copa y en la misma, se observa la migración de las setas JV1, JV2 y ZV2, característica de las especies del género Euseius, más un par de poros prominentes (Fig. 1). En la Figura 2, se observa la morfología de la espermateca, el cérvix es alargado en forma de letra "s", lo que coincide con lo descrito en las claves utilizadas.

En el género Euseius se tienen alrededor de 233 especies, aunque de ellas se han considerado solo 191 válidas (Demite et al. 2014), pero solo de muy pocas se han realizado estudios para evaluar su potencialidad como agentes de control biológico, lo que daría ventaja a los hallazgos taxonómicos, de unas especies sobre otras.

Por otra parte, se han realizado estudios ecológicos de fitoácaros en aguacateros de Michoacán y se ha considerado que la especie relacionada con $O$. persea es Euseius hibisci (Chant, 1959) (Salvador-de Jesús et al. 2016) sobre la base del estudio precedente de Estrada-Venegas et al. (2002).

Una explicación probable puede ser que $E$. consors desplazó a $E$. hibisci, lo cual es relativamente regular en agroecosistemas que reciben determinadas presiones antrópicas, que impactan a las especies presentes y que puede resultar en que dos especies con idénticos nichos ecológicos sujetas a la misma competencia por el alimento y el espacio, la coexistencia se pueda tornar imposible, con la eliminación de una de ellas (Fernández et al. 2007) o también, a cierta inexactitud en la identificación morfológica.

Esto último, es común en la actualidad, debido a que la capacidad de microscopía de que hoy se dispone, permite la observación de los caracteres con mayor calidad, que hace unos pocos años, lo que ha traído como resultado la re-identificación de los taxa presentes. Esto proporciona un valor particular a estos estudios, ya que la correcta identificación de las especies de agentes de control biológico es el punto

Tabla 1. Localidades de recolecta de Euseius consors (Acari: Phytoseiidae) en Michoacán, México.

\begin{tabular}{|c|c|c|c|}
\hline Municipio & Huerto & msnm & $\begin{array}{l}\text { Coordenadas geográficas } \\
\text { (Latitud/ Longitud) }\end{array}$ \\
\hline Tancítaro & La Vaina & 2080 & $19^{\circ} 17^{\prime} 11,6^{\prime \prime} \mathrm{N} 102^{\circ} 16^{\prime} 41,7^{\prime \prime} \mathrm{O}$ \\
\hline Nuevo Parangaricutiro & El Durazno & 2340 & $19^{\circ} 22^{\prime} 44,7^{\prime \prime} \mathrm{N} 102^{\circ} 14^{\prime} 25,1^{\prime \prime} \mathrm{O}$ \\
\hline Salvador Escalante & Cuitzitan & 2392 & $19^{\circ} 21^{\prime} 55,1^{\prime \prime} \mathrm{N} 101^{\circ} 39^{\prime} 10,5^{\prime \prime} \mathrm{O}$ \\
\hline Taretan & Mesa de San Ángel & 1539 & $19^{\circ} 23^{\prime} 00,1^{\prime \prime} \mathrm{N} 101^{\circ} 51^{\prime} 24,4^{\prime \prime} \mathrm{O}$ \\
\hline Uruapan & Cherangueran & 1934 & $19^{\circ} 28^{\prime} 01,5^{\prime} \mathrm{N} 102^{\circ} 05^{\prime} 13,1^{\prime \prime} \mathrm{O}$ \\
\hline Uruapan & $\begin{array}{l}\text { Campus Facultad de Agrobiología } \\
\text { "Presidente Juárez", UMSNH }\end{array}$ & 1630 & $19^{\circ} 23^{\prime} 41,1^{\prime \prime} \mathrm{N} 102^{\circ} 03^{\prime} 27,93^{\prime \prime O}$ \\
\hline Peribán de Ramos & "Champerico" & 1640 & $19^{\circ} 52^{\prime} 83,78^{\prime \prime} \mathrm{N} 102^{\circ} 42^{\prime} 63,7^{\prime \prime O}$ \\
\hline Comunidad Indígena J. Jesús Díaz Tzirio & "Centenario" & 2240 & $19^{\circ} 60^{\prime} 38,8^{\prime \prime} \mathrm{N} \mathrm{102} 33^{\circ} 80,5^{\prime \prime} \mathrm{O}$ \\
\hline
\end{tabular}




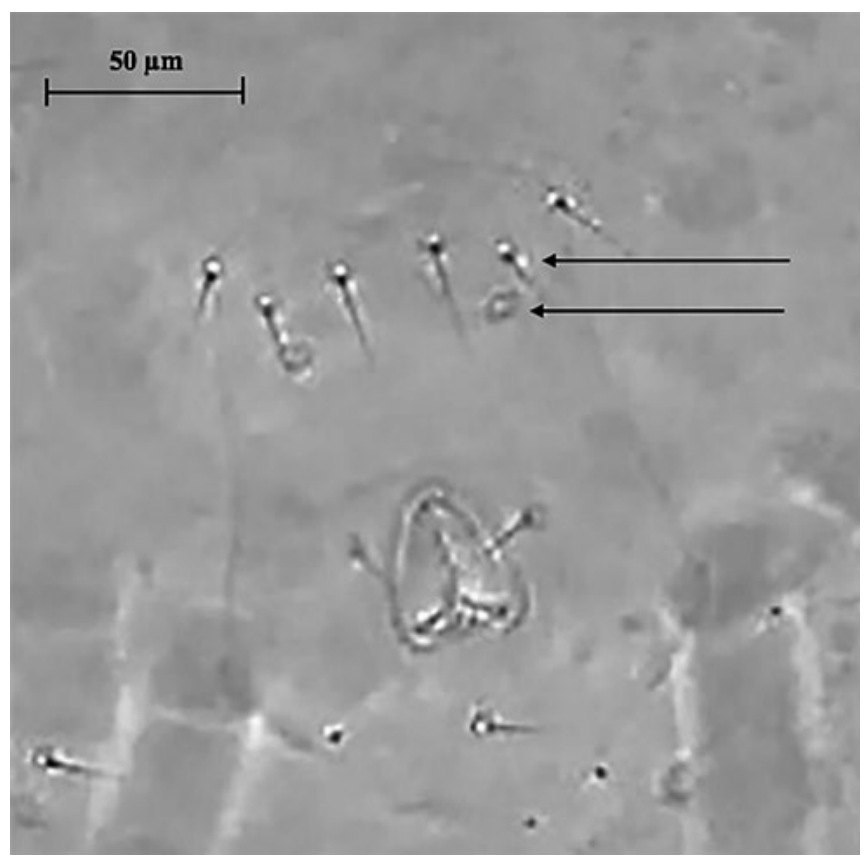

Figura 1. Placa ventrianal de Euseius consors mostrando las setas preanales y poros.

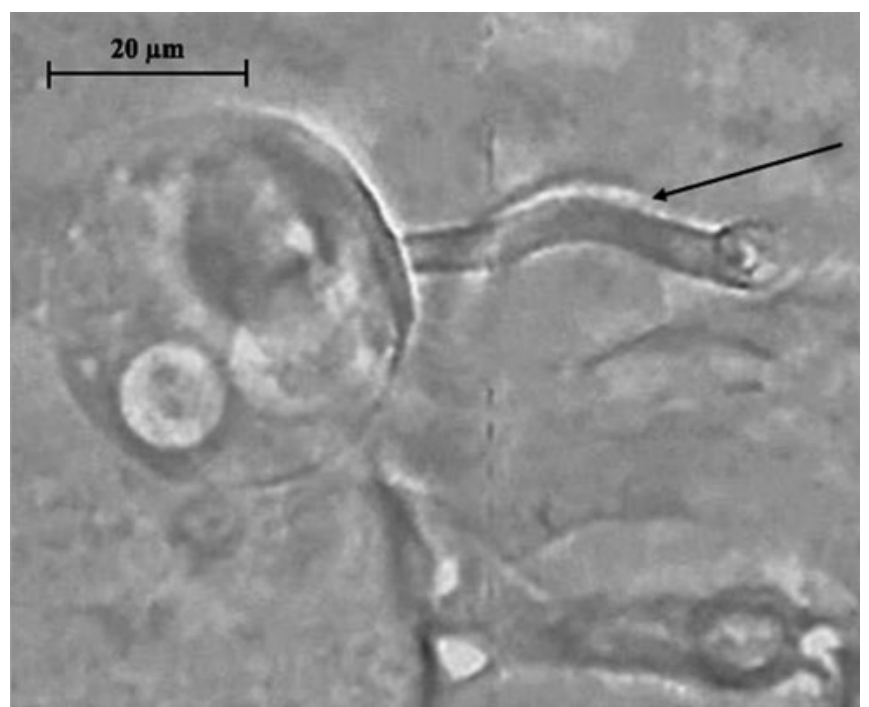

Figura 2. Espermateca de Euseius consors.

de partida y un requisito para evaluar su potencialidad en un agroecosistema. En la presente investigación se halló, de este género, a $E$. consors y este registro constituye la base para profundizar en los estudios biológicos y ecológicos de esta especie, así como en la continuidad de nuevas investigaciones. Con esto se considera la problemática fitosanitaria existente con los ácaros plaga en el cultivo del aguacate, la necesidad de transitar hacia métodos más amigables con el medio ambiente y de mayor sostenibilidad, así como el impacto económico y ecológico que tendría la aplicación de estos controles biológicos, en el principal cultivo de la región.

\section{Agradecimientos}

A la Coordinación de la Investigación Científica, UMSNH y al Tecnológico Nacional de México, TecNM por los apoyos para la ejecución de los proyectos.

\section{Literatura citada}

CHANT, D. A.; McMURTRY, J. A. 2005. A review of the subfamily Amblyseiinae Muma (Acari: Phytoseiidae): Part VI. The Tribe Euseiini n. tribe, subtribes Typhlodromalina n. subtribe, Euseiina n. subtribe, and Ricoseiina n. subtribe. International Journal of Acarology 31 (3): 187-224. https://doi. org/10.1080/01647950508684424

CORREDOR CAMARGO, E. S.; FONSECA CARREÑO, J. A.; PÁEZ BARÓN, E. M. 2012. Los servicios ecosistémicos de regulación tendencias e impacto en el bienestar humano. Revista de Investigación Agraria y Ambiental 3 (1): 77-83. https://doi. org/10.22490/21456453.936

DEMITE, P. R.; McMURTRY, J. A.; MORAES, G. J. 2014. Phytoseiidae Database: a website for taxonomic and distributional information on phytoseiid mites (Acari). Zootaxa 3795 (5): 571577. https://doi.org/10.11646/zootaxa.3795.5.6

EQUIHUA-MARTÍNEZ, A.; ESTRADA-VENEGAS, E. G.; GONZÁLEZ-HERNÁNDEZ, H. 2007. Plagas del Aguacate. pp. 133169. En: Téliz, D.; Mora, A. (Eds.). El Aguacate y su Manejo Integrado. 2da Edición. Mundi-Prensa. México S. A. de C. V. México, D.F., México 320 p.

ESTRADA-VENEGAS, E. G.; RODRÍGUEZ-NAVARRO, S.; McMURTRY, J. A. 2002. Some avocado mites from Michoacan México. International Journal of Acarology 28 (4): 387-393. https://doi.org/10.1080/01647950208684315

FERNÁNDEZ, M.; MIRANDA, I.; DÍAZ, M. E. 2007. Competencia de Diaphorina citri Kuwayama y Phyllocnistes citrella Stainton en el agroecosistema citrícola de la Isla de la Juventud, Cuba. Revista de Protección Vegetal 22 (1): 18-24. http://scielo.sld.cu/ scielo.php?pid=S1010-27522007000100002\&script=sci_arttext\&tlng=en

MORAES, G. J.; McMURTRY, J. A.; DENMARK, H. A; CAMPOS, C. B. 2004. A revised catalog of the mite family Phytoseiidae. Magnolia Press. Auckland, New Zealand, Zootaxa 434 (1): 1-494 https://doi.org/10.11646/zootaxa.434.1.1

PEÑA, J. ; WYSOKI, M. 2008. Plagas en Israel, México, Estados Unidos, Centroamérica y Perú. pp. 303-309. En: Ripa R. y Larral P. (Eds.). Manejo de Plagas en paltos y cítricos. Serie Documental: Colección Libros INIA - Instituto de Investigaciones Agropecuarias $\mathrm{N}^{\circ}$ 23. Ministerio de Agricultura, Chile. http:// www.avocadosource.com/books/ripa2008/ripa chapter_11b.pdf

SALVADOR-DE JESUS, L. A.; ESTRADA-VENEGAS, E. G.; EQUIHUA-MARTÍNEZ, A.; CHAIRES-GRIJALVA, M. P. 2016. Relación Oligonychus perseae (Prostigmata: Tetranychidae) y Euseius hibisci (Mesostigmata: Phytoseiidae) en dos huertas de aguacate Michoacán. Entomología Mexicana 3: 115119. http://www.socmexent.org/entomologia/revista/2016/AA/ Em\%20115-119.pdf

SERVICIO DE INFORMACIÓN AGROALIMENTARIA Y PESQUERA. 2021. Avance de Siembras y Cosechas. SIAP. Disponible en: https://nube.siap.gob.mx/avance_agricola/ [Fecha revisión 12 abril 2021].

\section{Origen y financiación}

La presente investigación forma parte del trabajo de dos proyectos de investigación, uno de ellos aprobado por el Consejo de posgrado de la Maestría en Agrobiotecnología y financiado por el Instituto Tecnológico Superior de Los Reyes y otro proyecto realizado en la Facultad de Agrobiología "Presidente Juárez”, Universidad Michoacana de San Nicolás de Hidalgo (UMSNH) por el CA 70, financiado por la Coordinación de la Investigación Científica, UMSNH, ambos en el estado de Michoacán, México; con el primer proyecto se titularon dos estudiantes y con el segundo uno. 


\section{Contribución de los autores}

Todos los autores aportaron en la elaboración del presente artículo y sus contribuciones en especifico fueron las siguientes: Mayra Ramos-Lima: coordinación general de uno de los proyectos, identificación de la especie, confección y revisión del manuscrito.

Margarita Vargas-Sandoval: coordinación general de uno de los proyectos, registro de datos en campo, revisión del manuscrito y apoyo en la fotografias.

José de Jesús Ayala-Ortega: colecta y revisión de muestras de campo, montaje y conservación de los especímenes colectados, análisis de la información.

Ma Blanca Nieves Lara-Chávez: corrección general del manuscrito de este artículo, apoyo financiero para su ejecución, Coordinadora del cuerpo académico CA-70, UMSNH. 